\title{
Carcinome épidermoïde cutané métastatique zostériforme
}

\author{
Gabriel Crevier-Sorbo MD MSc, Aura Cernii MD, Andrei Cepoi MD
}

— Citation : CMAJ 2021 October 4;193:E1536. doi : 10.1503/cmaj.210551-f

Voir la version anglaise de l'article ici : www.cmaj.ca/lookup/doi/10.1503/cmaj.210551

$\mathbf{U}$ n homme de 59 ans a consulté aux urgences pour un érythème cutané extrêmement douloureux accompagné de papules, d'ulcérations, de croûtes et d'une lymphadénopathie axillaire, présent depuis 1 mois. L'érythème s'étendait de son aisselle droite à sa ligne médiane, le long des dermatomes T3 et T4 (figure 1). Il avait été traité sans succès au moyen d'un cycle long d'acyclovir oral et de céfalexine pour ce que l'on croyait être un zona surinfecté. Ses antécédents médicaux étaient sans particularité, à l'exception de l'amputation de l'annulaire droit 6 mois auparavant pour un carcinome épidermoïde cutané qui avait envahi sa phalange distale. Il était immunocompétent et avait subi une tomographie par émission de positrons du corps entier 1 mois avant l'apparition de l'érythème, sans résultat anormal.

Nous avons prélevé un échantillon de lésion cutanée par biopsie, et l'examen anatomopathologique a révélé la présence d'un carcinome épidermoïde mal différencié, avec envahissement périneural et infiltration des tissus sous-cutanés. Nous avons posé un diagnostic de carcinome épidermoïde cutané métastatique zostériforme et avons envoyé le patient en chimiothérapie et en radiothérapie palliatives. Il est décédé 1 mois plus tard, à la suite de la progression fulgurante de son cancer.

Les carcinomes épidermoïdes cutanés sont habituellement traités par exérèse chirurgicale et moins de $4 \%$ deviennent métastatiques ${ }^{1}$. Les facteurs de risque à l'égard de la maladie métastatique sont : sexe masculin, faible statut socioéconomique, immunosuppression et âge supérieur à 80 ans $^{1}$. Un diamètre tumoral de plus de $2,0 \mathrm{~cm}$, l'envahissement au-delà des graisses sous-cutanées,

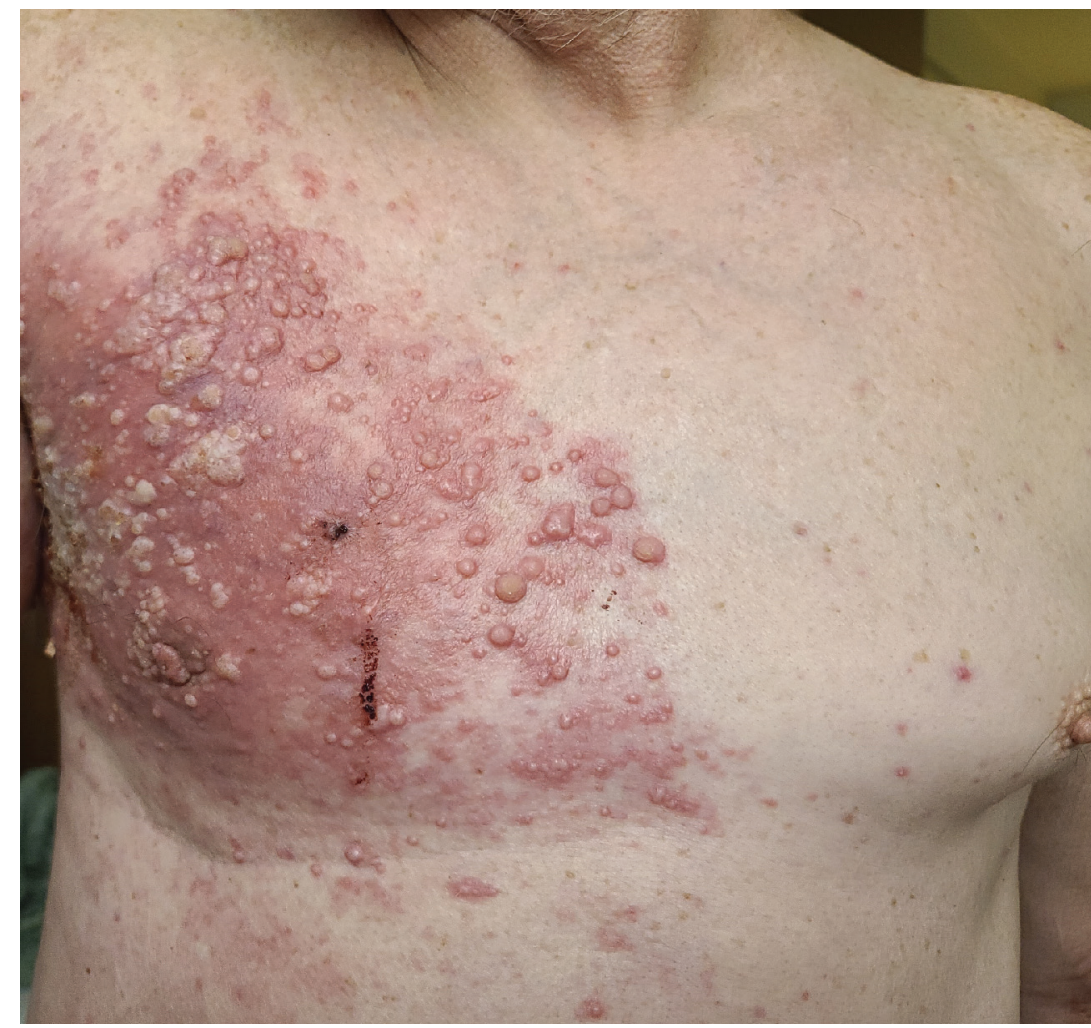

Figure 1 : Photographie d'un homme de 59 ans atteint d'un carcinome épidermoïde cutané métastatique, montrant un érythème accompagné de papules et d'ulcérations distribuées le long des dermatomes T3 et T4.

l'envahissement périneural, l'érosion osseuse, le sous-type desmoplastique et la piètre différenciation tumorale sont associés à une propagation lymphatique et à des métastases distales ${ }^{2}$.

Le zona, ou herpès zoster, se manifeste souvent par un érythème extrêmement sensible au niveau de certains dermatomes, avec des vésicules regroupées sur un fond érythémateux, et peut durer 3-4 semaines. Un carcinome épidermoïde cutané métastatique zostériforme peut ressembler 
étroitement au zona et est également extrêmement douloureux. Il est rare et survient probablement par propagation lymphangitique rétrograde des cellules tumorales; le pronostic est extrêmement sombre ${ }^{3-6}$. Le diagnostic repose sur un fort degré de suspicion chez les patients qui ont des antécédents de cancer de la peau ou d'autres cancers et qui manifestent un érythème de type zostérien réfractaire aux antiviraux $x^{4,5}$.

\section{Références}

1. Venables ZC, Autier P, Nijsten T, et al. Nationwide incidence of metastatic cutaneous squamous cell carcinoma in England. JAMA Dermatol 2019;155:298-306.

2. Que SKT, Zwald FO, Schmults CD. Cutaneous squamous cell carcinoma: incidence, risk factors, diagnosis, and staging. J Am Acad Dermatol 2018;78:237-47.

3. Savoia P, Fava P, Deboli T, et al. Zosteriform cutaneous metastases: a literature meta-analysis and a clinical report of three melanoma cases. Dermatol Surg 2009;35:1355-63.

4. Maki Y, Kimizuka Y, Murakami K, et al. Zosteriform skin metastasis caused by retrograde lymphatic migration of metastatic squamous cell lung carcinoma. BMC Pulm Med 2021;21:41.

5. Kikuchi Y, Matsuyama A, Nomura K. Zosteriform metastatic skin cancer: report of three cases and review of the literature. Dermatology 2001;202:336-8.

6. Williams DC, Livingston R. High aggressive herpetiform squamous cell carcinoma. Plast Reconstr Surg Glob Open 2015;3:e522.
Intérêts concurrents : Aucun déclaré.

Cet article a été évalué par des pairs.

Les auteurs ont obtenu le consentement du patient.

Affiliation : Département de médecine familiale, Université du Vermont - Champlain Valley Physicians Hospital, Plattsburgh, NY.

Propriété intellectuelle du contenu : Il s'agit d'un article en libre accès distribué conformément aux modalités de la licence Creative Commons Attribution (CC BY-NC-ND 4.0), qui permet l'utilisation, la diffusion et la reproduction dans tout médium à la condition que la publication originale soit adéquatement citée, que l'utilisation se fasse à des fins non commerciales (c.-à-d., recherche ou éducation) et qu'aucune modification ni adaptation n'y soit apportée. Voir : https://creativecommons.org/licenses/by -nc-nd/4.0/deed.fr.

Correspondance : Gabriel Crevier-Sorbo, gcrevier-sorbo@cvph.org

Les images cliniques sont choisies pour leur caractère particulièrement intéressant, classique ou impressionnant. Toute soumission d'image de haute résolution claire et bien identifiée doit être accompagnée d'une légende aux fins de publication. On demande aussi une brève explication (300 mots maximum) de la portée éducative des images, et des références minimales. Le consentement écrit du patient au regard de la publication doit être obtenu avant la soumission. 